\title{
A Revista Brasileira de Hematologia e Hemoterapia na coleção SciELO - Brasil
}

\author{
Milton A. Ruiz
}

\begin{abstract}
A Revista Brasileira de Hematologia e Hemoterapia foi inserida na base de dados da Scientific Electronic Library On-Line (SciELO) em outubro de 2001. Esta conquista propicia o relato da evolução do veículo de divulgação da Sociedade Brasileira de Hematologia e Hemoterapia que se iniciou em 1973. Até 1984 o Boletim foi um veículo de divulgação, transformando-se em seguida em revista científica. Em 1999 mudou de título, modernizou-se e passou a ser também o órgão oficial da Sociedade Brasileira de Transplante de Medula Óssea.

Para o entendimento da referida conquista foi realizado um histórico sobre $o$ Institute for Scientific Information (ISI), principal base de dados internacional, e a SCIELO, base de dados nacional de recuperação de linha. Ao final o autor apresenta uma reflexão sobre o futuro da RBHH e os caminhos que o periódico deve trilhar com a finalidade de aumentar a sua importância no meio científico.

Rev.bras.hematol.hemoter.,2002,24(1): 3-7
\end{abstract}

Palavras-chave: Periódicos científicos, base de dados, SciELO

\section{Introdução}

Em reunião de 23 de outubro de 2001 no II Encontro do Comitê Consultivo da Scientific Eletronic Library On-Line (SCiELO), a Revista Brasileira de Hematologia e Hemoterapia (RBHH) foi indexada em sua base de dados (1). Na ocasião, após processo de avaliação de 102 periódicos, foram aprovados para inserção nesta base de dados 32\% dos títulos analisados. $\mathrm{Na}$ área biológica dos 55 periódicos avaliados, somente 15 foram aprovados e dentre os mesmos a RBHH (1).

Esta conquista nos obriga a apresentar à comunidade hematológica um relato da evolução de nosso periódico científico, discutir sobre base de dados e refletir sobre o futuro e os caminhos que a revista deve trilhar para o seu progresso. Após este preâmbulo, considero relevante e pertinente um breve retorno ao passado com a finalidade de não esquecer os caminhos que levaram a RBHH até o ponto em que se encontra.

A RBHH é sucedânea do Boletim da Sociedade Brasileira de Hematologia (BSBHH), que circulou no período de 1973 a 1998. Em 1973, com o ressurgimento da Sociedade Brasileira de Hematologia e Hemoterapia (SBHH), foi criado O Boletim, que mensalmente passou a contar a história da hematologia e hemoterapia. Além de divulgar as atividades da SBHH, o informativo exercia forte pressão política aos órgãos governamentais e tinha como linha mestra e política a defesa da especialidade.

O Boletim espelhou e divulgou em suas páginas, a luta da especialidade na implantação

Prof. Adjunto, Departamento de Clínica Médica da Faculdade de Medicina de São José do Rio Preto , SP Coordenador da Unidade de Transplante de Medula Óssea do Hospital de Base São José do Rio Preto - FUNFARME Editor da Revista Brasileira de Hematologia e Hemoterapia 
de vários programas nacionais e de interesse público, dentre eles o da doação voluntária e a proibição da remuneração ao doador de sangue, prática disseminada então em nosso país. A partir de 1984, por orientação da diretoria da época, de veículo de divulgação O Boletim transformouse em revista científica, passando então a ser denominado "Boletim da Sociedade Brasileira de Hematologia e Hemoterapia" (BSBHH), com International Standard Serial Number (ISSN) de 0102-7662 no cadastro do Instituto Brasileiro de Informação em Ciência e Tecnologia (IBICT).

Após dois anos de circulação, a revista foi indexada no antigo Index Médico Latino Americano (IMLA) e, posteriormente, também no Compact Disk Read Only Memory (CD rom) da Literatura Latino-Americana e do Caribe de Ciências da Saúde (LILACs) (2). O BSBHH esteve presente no meio científico até 1998 com 179 fascículos distribuídos em 20 volumes publicados (2).

Atendendo à necessidade de ampliar os horizontes, o BSBHH, a pedido, foi avaliado e sofreu uma análise crítica de especialista no tema (3), recebendo sugestões de modificações. Dentre elas, sugestões em relação ao formato, conteúdo, divulgação, circulação e alertado sobre a ausência do periódico no acervo das principais bibliotecas institucionais do país. Assim, modificações no periódico científico foram sendo realizadas junto à mudança do título para Revista Brasileira de Hematologia e Hemoterapia, ISSN 1516-8484.

Com a medida, houve modificação exclusiva do título sem perda de ligações com o passado, sendo mantida inclusive a continuidade do veículo no número progressivo de volumes editados.

Esta desta medida deveu-se à perspectiva de albergar no futuro subáreas da hematologia, pois com o título de BSBBH esta possibilidade estava excluída. A modificação rendeu frutos e no ano 2000, a partir do volume 22, a revista tornou-se também o órgão oficial da Sociedade Brasileira de Transplante de Medula Óssea, fato este que propiciou um incremento de qualidade e um aumento da representatividade da $\mathrm{RBHH}$ na hematologia brasileira (4).

Desde a modificação do título, a RBHH solicitou sua inserção na coleção Brasil da Scientific Eletronic Library On-Line (SciELO) (4). O SciELO é uma base dados on-line ou de recuperação de linha (5). O mesmo é o resultado de projeto de parceria entre a Fundação de Amparo a Pesquisa do Estado de São Paulo (Fapesp) e o Centro Latino-Americano e do Caribe de Informação em Ciências da Saúde (Bireme), tendo o objetivo de albergar e disponibilizar eletronicamente as publicações científicas do país e da América latina (5-6). Organiza e publica textos completos das revistas escolhidas segundo critérios de qualidade, que estão disponíveis na internet em seu site, e publica indicadores de uso e impacto (5).

O principal objetivo do projeto, iniciado em 1997, é o de aumentar a visibilidade da produção científica brasileira criando mecanismos complementares aos do ISI (6). Visa também desenvolver uma metodologia de publicação eletrônica com o objetivo de complementar o registro bibliográfico e indexação da Literatura Latino-Americana e do Caribe em Ciências da Saúde (Lilacs). O projeto teve o seu início efetivo com 10 periódicos e apoio dos seus respectivos editores científicos que participaram de implantação da metodologia SciELO e os mesmos por motivos históricos estão listados abaixo (tabela 1) (5). Atualmente, no seu site, estão disponíveis 70 títulos de periódicos de várias áreas da ciência.

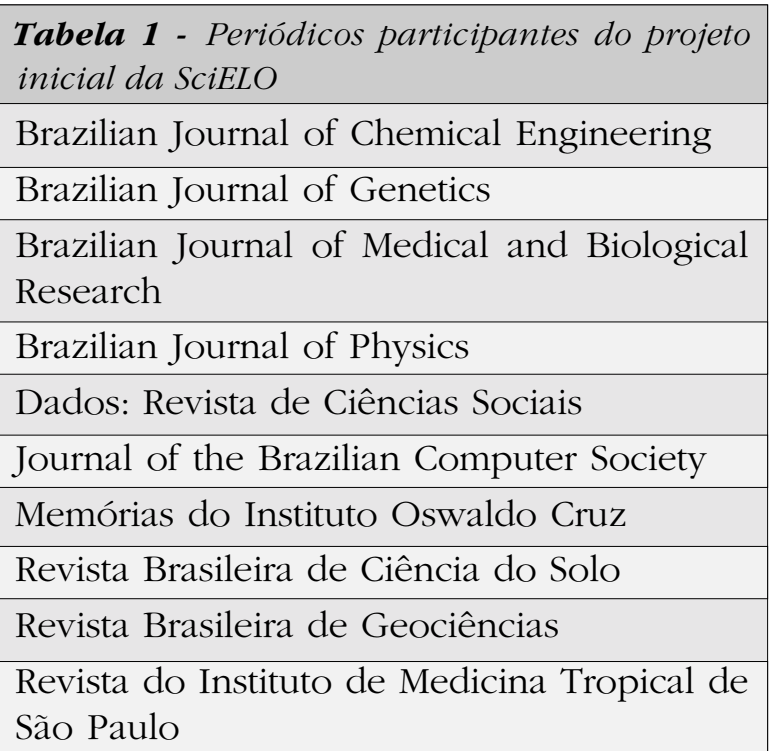

Tabela 1 - Periódicos participantes do projeto cial da SciELO

Brazilian Journal of Chemical Engineering

Brazilian Journal of Medical and Biological Research

Journal of the Brazilian Computer Society

Memórias do Instituto Oswaldo Cruz

Revista Brasileira de Geociências

São Paulo 


\section{Bases de Dados}

Desde o aparecimento do Journal des Sçavants na França e do Royal Philosophical Transactions na Inglaterra, em 1665, os periódicos científicos regulares passaram a desempenhar papel essencial de comunicação entre os pesquisadores (7).

Os periódicos se organizaram e os trabalhos de precursores começaram a servir de base aos futuros pesquisadores na construção de suas obras e informação nas listas de citações. Logo com o avanço do conhecimento, da multiplicidade e velocidade de novas informações, emergiu a necessidade de análise do mérito dos trabalhos que eram apresentados para serem publicados. Os periódicos criaram então a figura do revisor ou árbitro, oriundo de seus próprios pares, para exercer esta função.

Esta medida, que é uma regra, era particular no início em cada periódico, e atualmente salvo exceções à maioria dos veículos de comunicação científica de nossa área, inclusive a RBHH, segue as orientações e as condutas que estão preconizadas pelo International Committee of Scientific Editors (8).

O grupo de Vancouver, como é denominado o Comitê, além de orientar em relação ao formato de envio e de apresentação dos artigos, sugere políticas editoriais internas no sentido de preservar a independência do editor e do seu corpo editorial com a finalidade de estimular o progresso e manter a lisura científica no desenvolvimento do processo editorial (8).

Nas publicações, os cientistas mostram como construíram seus trabalhos e demonstram que seus relatos estão escudados em obras anteriores, mencionando-as em seus textos e em uma lista de referência.

O número de publicações de um grupo de pesquisa é considerado um indicador de produção científica. O número de vezes em que uma ou mais obras de um determinado grupo é citado em outras publicações, tem importância na mensuração do impacto e da visibilidade científica das obras dos autores deste grupo. Assim, a publicação e a mensuração de suas citações são imprescindíveis como método de avaliação científica e citação um mérito para o autor e seu grupo.

Garfield, em 1972, em seu artigo sobre análise das citações como método de avaliação de periódicos, discorre sobre como foram introduzidas e a importância das bases de dados na avaliação científica $(9,10,11)$. Define-se como base de dados o conjunto organizado de informações ou de documentos, ou seja, ele é a base do pensamento especializado (12). O seu desenvolvimento remonta a 1927, quando Gross em seu artigo no Journal of the American Chemical Society avaliou as listas de referências das publicações de um ano do referido periódico (9).

A base de dados de maior repercussão internacional é a do Institute for Scientific Information (ISI), criado em 1958 (12). O ISI é o responsável pelo Science Citation Index (SCI), considerado o único índice editado de ciência e que abrange 5.600 títulos de periódicos $(9$, 10, 11, 12).

O Institute for Scientific Information com o Science Citation Index cobre a maioria das revistas internacionais importantes na área da ciência pura, aplicadas e médicas (12).

No sistema global de revistas, o ISI - através de monitorização constante - distingue as revistas centrais ou essenciais e as periféricas, em geral revistas voltadas para interesses regionais (13).

A participação das revistas nacionais nesta base de dados é pequena e até recentemente somente 17 periódicos nacionais encontravamse indexados no ISI (12).

No Brasil, a dificuldade de produzir uma revista científica é imensa e esta dificuldade advém do passado e decorre mesmo da tardia introdução da tipografia e o início das publicações no país. A imprensa, como a conhecemos, só aportou aqui em 1808 em decorrência da vinda da família real portuguesa para o Brasil. Falta de tradição, barreira da língua, poucos recursos financeiros e ausência de uma política de divulgação da produção científica nacional, são considerados fatores para o atraso e responsável pela pouca importância dos periódicos nacionais no meio científico internacional. 
Em que pese existirem indicadores recentes mais animadores quanto à produção científica nacional, a mesma ainda é considerada insípida internacionalmente (6). Os periódicos científicos, de importância considerável no nosso meio, como os das Sociedades Médicas Brasileiras, estão na sua maioria na marginalidade científica, pois não são referidas e apresentam dificuldades de pontualidade na periodicidade, de qualidade do material publicado e, principalmente, lutam com a falta de infra-estrutura.

Por estes e vários outros motivos, muitos dos periódicos nacionais não resistem e poucos conseguem se manter após alguns fascículos e aos primeiros anos de entusiasmo.

\section{Discussão}

Após discorrer sobre as bases de dados e enfatizar a importância destes registros na manutenção e divulgação do produto científico, consideramos importante uma reflexão sobre a $\mathrm{RBHH}$. A revista, em que pese a conquista obtida com a inserção na base de dados SciELO, ela encontra-se na marginalidade científica. Podemos afirmar esta assertiva se seguirmos os parâmetros da ISI, de que não fazer parte do seu núcleo essencial de revistas da área ou não estar indexada em sua base dados, configura um demérito.

Para justificar a ausência dos periódicos nacionais no ISI existem inúmeras alternativas. O formato, a pontualidade, a língua, tendo sido este um dos critérios para exclusão de um periódico brasileiro de sua base dados foi o de que o mesmo publicava artigos em português (14). No entanto, em relação à língua, os critérios do ISI são ambíguos, visto que inclusive algumas publicações nacionais que continuam nesta base de dados, publicam em português. Assim, no futuro, apesar da RBHH ser bilíngüe, a opção de publicação da revista ou da edição de fascículos exclusivamente em inglês deverá ser discutida.

Outro fator de exclusão dos periódicos das bases de dados é a impontualidade na periodicidade. Há três anos a RBHH superou este problema, pois a periodicidade tem sido mantida, fato este que certamente pesou na sua avaliação.

Em relação à periodicidade da $\mathrm{RBHH}$, que era quadrimestral oficialmente, na realidade quatro ou cinco fascículos anuais compõem o nosso acervo, devido à edição de fascículos especiais. Seguindo os critérios e recomendação da Scielo, a revista deverá circular trimestralmente (15). Em relação ao conteúdo, que considero modesto, ele deve ser incrementado e o número de contribuições obrigatoriamente precisa aumentar para atender aos critérios mínimos de comunicações anuais que teremos de atingir (15).

Considero importante que os grupos nacionais participem mais ativamente e sua colaboração não se restrinja a relatos de casos e às contribuições relevantes oriundas do produto de pesquisas das teses referidas em nossas páginas, e que outras não referidas nos sejam encaminhadas para entrar no acervo científico nacional e de preferência na RBHH.

Este é um trabalho árduo a ser vencido, o de convencer a todos de que o artigo publicado no Brasil também tem seu valor. A SciELO com o seu projeto visa isto, pois as revistas de sua coleção são monitorizadas com a mesma metodologia e as revista inseridas na sua base também tem o seu fator de impacto. Como já foi descrito, a SciELO visa complementar o ISI responsável pelo fator de impacto, considerado divisor de águas no mercado mundial de publicação científica $(14,16)$. Assim, espero que o fluxo de contribuições seja incrementado por estarmos no mesmo nível de outras publicações nacionais e a partir de agora o desafio de manter a RBHH na base de dados. A busca de inserção em outras bases de dados será outro objetivo, pois aumentará a possibilidade de ser referida.

No universo científico a ordem é: publique ou pereça, seja citado ou desapareça (16) e, para tanto, para o futuro da RBHH é vital que a citação de suas obras ocorra tanto em suas páginas como nos outros periódicos.

Finalizando, a hematologia brasileira e as sociedades que a RBHH representa, para este ano têm um desafio à sua frente, que é o de preencher os critérios da SciELO para se manter em sua base de dados. 
Brazilian Journal of Hematology and Hemotherapy in SciELO - Brazil

Milton A. Ruiz

\section{Abstract}

The Brazilian Hematology and Hemotherapy Journal (Revista Brasileira de Hematologia e Hemoterapia) was included in the data base of Scientific Electronic library on-line (SciELO) in October of 2001.

This achievement encourages a bistoric registration of the means by which the performance of the Brazilian Hematology and Hemotherapy Society, which began in 1973, has been divulged.

Until 1984, the Boletim was the instrument of notification of the work of the society which soon became a scientific journal. In 1999, the title was changed and the journal was modernized. At this time it also became the official publication of the Brazilian Society of Bone Marrow Transplantation.

To better understand the importance of this victory, a historic review of the Institute for Scientific Information (ISI), the main international data base and SCIELO, the national data base was assembled.

Finally the author reflects on the future of the Journal and the paths that it should follow with the objective of increasing the periodicals importance in the scientific world.

Rev.bras.hematol.hemoter.,2002,24(1): 3-7

Keywords : Scientific Periodical; Data Base; SCiELO

\section{Referências Bibliográficas}

1. SciELO. www.scielo.br/avaliacao/ avaliacao_en.htm acesso em 12/02/02.

2. Ruiz MA, Fragoso YD. Boletim da Sociedade Brasileira de Hematologia e Hemoterapia. Rev.bras.hematol hemoter. 1999. 21(01): 03-06.

3. Kryzyzanowski RF. Análise crítica do Boletim da Sociedade Brasileira de
Hematologia e Hemoterapia comunicação pessoal 1998

4. Ruiz MA. O volume 22 e as perspectivas futuras. Rev.bras.hematol.hemot. 2000; 22(3): 360.

5. Packer AL, Biojone MR, Antonio I, Takenaka RM, Garcia AP, Costa da Silva A, Murasaki RT, Mylek C, Reis OC, Delbucio HCRF. SciELO: uma metodologia para publicação eletrônica. Ci.Inf. 1998 ; 27 (2): 109-121.

6. Meneghini R. Avaliação da produção científica e o Projeto SciELO. Ci.Inf. 1998; 27 (2): 219-220.

7. Pessanha C. Critérios editoriais de avaliação científica: notas para discussão. Ci. Inf. 1998; 27 (2): 226-229.

8. International Committee of Medical Editors. Uniform requirements for manuscripts submitted to biomedical journals. Ann.Intern.Med. 1997; 126 (1):36-47.

9. Garfield E. Citation Analysis as a tool in journal evaluation. Science. 1972. 178 (4060): 471-479.

10. Garfield E. Citation indexes for science. Science. 1955. 122: 188.

11. Garfield E. Science citation index a new dimension in indexing. Science. 1964. 144: 649 .

12. Targino MG, Garcia JCR. Ciência brasileira na base de dados do Institute for Scientific Information (ISI). Ci.Inf. 2000, 29 (1):103-117.

13. Testa J . A base de dados ISI e seu processo de seleção de revistas. Ci.Inf. 1998; 27(2): 233-235.

14. Gomes SP, Santos MALC. Avaliação de um periódico na área de medicina tropical. Ci.Inf. 2001, 30 (2): 91-100.

15. Criteria SciELO Brazil, www.scielo.br/ criteria/scielo_brasil_en.html.

16. Forattini OP. A tríade da publicação científica. Rev.Saúde Pub. 1996, 30 (1) 3-12.

Recebido:

Aceito: 